\section{GG-02 APOLIPOPROTEIN L1 RISK VARIANTS ASSOCIATE WITH INCREASED SLE DAMAGE INDEPENDENT OF INFLAMMATION IN GHANAIAN SLE PATIENTS}

${ }^{1}$ Ashira Blazer*, ${ }^{2}$ Ida Dzifa Dey, ${ }^{2}$ Margret Reynolds, ${ }^{2}$ Festus Ankrah, ${ }^{1}$ Jill Buyon, ${ }^{1}$ Robert Clancy. ${ }^{1}$ New York University School of Medicine, New York, NY; ${ }^{2}$ Korle bu Teaching Hospital, Accra Ghana

\subsection{6/lupus-2018-Ism.89}

Background The two Apolipoprotein L1 (APOL1) risk variants (RV), G1 and G2, are enriched in African populations due to resistance to Trypanosoma brucei, a parasite endemic in West Africa. This improved fitness comes with the cost of propensity toward renal disease by multiple causes including SLE. In Ghana, up to $60 \%$ of individuals are heterozygous and $30 \%$ are variant homozygous. However, APOL1 variant phenotypes have never been described in a Ghanaian SLE cohort. Further it remains unclear whether RV associated nephritis progression is driven by accelerated inflammation or intrinsic renal disease. Accordingly, we evaluated APOL1 genotype-phenotype traits in the context of SLE activity in 101 Ghanaian patients seeking care at Korle bu Teaching Hospital in Accra, Ghana.

Methods 101 Ghanaian patients meeting at least 4 clinical criteria for SLE were stratified by APOL1 genotype PCR/ sequencing as follows: ancestral (G0/G0), RV heterozygotes (RV/G0), and RV homozygotes (RV/RV). DNA was extracted from saliva. Clinical endpoints including demographics, ACR criteria, SLEDAI score, and SLICC damage index were recorded at time of blood draw. Based on cytokines known to fluctuate with SLE activity in African Americans, a 12 cytokine array was performed on sera to determine active inflammatory pathways. Interferon signature as measured by the WISH cell assay was completed.

Results The frequencies of the G0, G1, and G2 alleles were $63.3 \%, 24.2 \%$, and $12.5 \%$ respectively. Subjects were $100 \%$ female, and 32.1 years of age with a disease duration of 2.9 years. There were no differences in demographics across the genotypes. The RV associated with higher BP: 107.4/72.3, 108.5/72.1, and 120.6/83.4 in the G0/G0, RV/G0, and RV/RV groups respectively (p: 0.013 systolic; 0.003 diastolic). Among those with nephritis, RV/RV associated with increased creatinine and proteinuria with creatinine values of $0.9,1.1$, and 2.5, and average (3 visit) urine dipstick values of 1.0, 1.2, and 3.33 in the $\mathrm{G} 0 / \mathrm{G} 0, \mathrm{RV} / \mathrm{G} 0$, and $\mathrm{RV} / \mathrm{RV}$ groups respectively (creatinine: $p=0.03$; urine dipstick $p=0.01$ ). $R V / R V$ carriers were on higher doses of prednisolone $(17.2 \mathrm{mg}$ ) compared to G0/G0 or RV/G0 carriers (11.2 mg; $11.8 \mathrm{mg}$ respectively). SLEDAI scores were comparable across the genotypes, however RV/RV associated with elevated SLICC damage index: G0/G0 or RV/G0: 0.95 vs RV/RV: 1.7; driven by renal, CVD, and neurologic manifestations G0/G0: 0.46, RV/G0: $0.39, \mathrm{RV} / \mathrm{RV}: 1.25 ; \mathrm{p}=0.03$ Despite more damage, $\mathrm{RV} / \mathrm{RV}$ individuals had lower dsDNA titers $(10.7 \mathrm{IU} / \mathrm{mL})$ than $\mathrm{G} 0 / \mathrm{G} 0$ (57.1 IU/mL) and RV/G0 (95.6 IU/mL) carriers (p: 0.03). Cytokine scores were also lower in RV/RV carriers compared to G0/G0 and RV/RV, for example serum IL8 levels were $34.6 \mathrm{pg}, 33.2 \mathrm{pg}$, and $13.8 \mathrm{pg}$ (IL-8: $\mathrm{p}=0.04$ ). Both G0/G0 and RV/G0 individuals exhibited more elevated IFN signatures than RV/RV individuals $(245.7,81.3$, and 24.3 respectively, $\mathrm{p}: 0.04)$.

Conclusions Taken together, the APOL1 RV associated with increased blood pressure, creatinine, proteinuria, and SLICC damage score in this Ghanaian SLE cohort. Paradoxically, RV homozygotes exhibited lower dsDNA titers and lower cytokine profiles than G0/G0 or RV/G0 patients. This suggests that RV homozygotes may have an intrinsic propensity toward organ damage independent of SLE activity.

\section{GG-03 IRF5 GENETIC RISK VARIANTS DRIVE AN SLE IMMUNE- PHENOTYPE IN HEALTHY DONORS}

${ }^{1}$ Dan Li, ${ }^{1}$ Bharati Matta, ${ }^{1}$ Su Song, ${ }^{2}$ Kim Simpfendorfer, ${ }^{2}$ Peter Gregersen, ${ }^{1}$ Betsy J Barnes* . ${ }^{1}$ Center for Autoimmune, Musculoskeletal and Hematopoietic Diseases, The Feinstein Institute for Medical Research, Manhasset, NY; ${ }^{2}$ Center for Genomics and Human Genetics, The Feinstein Institute for Medical Research, Manhasset, NY

\subsection{6/lupus-2018-Ism.90}

Background Gene expression profiling has provided significant insight into SLE pathology through the identification of an IFN gene signature, a neutrophil-specific signature, and a plasma cell signature that correlate with disease activity. While genetic variants have also been shown to correlate with disease activity, the mechanism(s) by which they confer risk to SLE are not fully elucidated. Genetic variants within or near the interferon regulatory factor 5 (IRF5) locus associate with SLE across ancestral groups. Four IRF5 variants have been identified that strongly associate with SLE risk and make up the major risk haplotype in European Caucasians (rs2004640, rs10954213, rs10488631, and rs142738614 (CGGGG indel)). To determine how these variants contribute to SLE risk, we performed global immune-phenotyping on healthy donors homozygous for the major risk and non-risk haplotypes.

Methods Healthy donors (no personal/family history of autoimmune/inflammatory diseases or cancer) are participants of the Feinstein Genotype and Phenotype $(\mathrm{GaP})$ Registry that is genotyped on the Illumina Human Immunochip. Individual donors carrying the homozygous risk and non-risk haplotype were called in a minimum of 4 times over a 2 year-period for independent blood draws. Lymphoid and myeloid subsets were analyzed by flow cytometry on a BD Fortessa. Serum autoantibodies were measured by ANA-Hep-2, ELISA and a multiplex antibody profiling array. IRF5 expression and activation were determined on an Amnis Imagestream X Mark II imaging flow cytometer. B cell differentiation was determined by in vitro culture. NETosis was determined by fluorescent microscopy and flow cytometry. Ex vivo co-culture was used to identify antigenic triggers in risk and non-risk donors that drive B cell differentiation.

Results We observed a striking homogeneity within haplotype groups that was conserved over time and could be replicated by ex vivo co-culture. We found that the IRF5 risk haplotype confers cell lineage-specific changes that mimic pre-symptomatic SLE. Interestingly, and contrary to what has been previously reported in B lymphoblastoid cell lines and SLE immune cells, IRF5 genetic variants had no effect on the overall levels of IRF5 expression in cells from healthy donors. Instead, IRF5 risk carriers had elevated numbers of circulating plasmacytoid dendritic cells and plasma cells, positive ANA, constitutive IRF5 hyper-activation, and spontaneous NETosis.

Conclusions Data suggest that individuals carrying IRF5-SLE risk haplotypes are particularly susceptible to environmental or stochastic influences that can trigger a transition to chronic immune activation, which in turn predisposes to the development of clinical lupus. 